\title{
Aesthetic Correction of Severe Facial Asymmetry in a Deformational Plagiocephaly Patient: A Case Report and Literature Review
}

\section{Jae Yeon Park, Hyo Joong Kim, Seil Lee, Sung Gyun Jung}

Department of Plastic and Reconstructive Surgery, National Medical Center, Seoul, Korea
No potential conflict of interest relevant to this article was reported.
Deformational plagiocephaly (DP) (also referred to as positional plagiocephaly) has long posed challenges for plastic surgeons because it is difficult to differentiate from several other diseases, such as unilateral coronal synostosis, hemifacial microsomia, and unilateral lambdoidal craniosynostosis. These diseases can actually masquerade as DP or vice versa. Only in recent years has the differential diagnosis among these diseases become possible through improved imaging modalities, such as computed tomography, and a greater understanding of their pathophysiology. Herein, we report a rather rare, yet severe, form of DP that can easily be confused with the aforementioned diseases.

Keywords Blepharoplasty, Facial asymmetry, Nonsynostotic, Plagiocephaly

\section{INTRODUCTION}

Deformational plagiocephaly (DP), which is interchangeably referred to as positional plagiocephaly, is an acquired disease that mimics several congenital malformations such as hemifacial microsomia, unilateral coronal craniosynostosis, and unilateral lambdoidal craniosynostosis. Only in the last several decades has this disease been diagnosed with more objectivity. Before an adequate amount of data was accumulated to allow a sufficiently deep understanding of this disease entity, even plastic surgeons had difficulty making a precise diagnosis and many babies were misdiagnosed with craniosynostosis or hemifacial microsomia, only to undergo unnecessary surgery. In many instances of DP, the facial features are mild, obviating the need for surgery. Here we report a rather rare, yet severe, case of DP in a 22 -year-old female patient.

Received: Jul 24, 2017 Revised: Sep 18, 2017 Accepted: Sep 23, 2017 Correspondence: Seil Lee Department of Plastic and Reconstructive Surgery, National Medical Center, 245 Eulji-ro, Jung-gu, Seoul 04564, Korea. E-mail: leeseil.nmcps@gmail.com

Copyright @ 2017 The Korean Society for Aesthetic Plastic Surgery.

This is an Open Access article distributed under the terms of the Creative Commons Attribution Non-Commercial License (http://creativecommons.org/licenses/by-nc/4.0/) which permits unrestricted non-commercial use, distribution, and reproduction in any medium, provided the original work is properly cited. www.e-aaps.org

\section{CASE REPORT}

A 22-year-old female patient visited our outpatient department complaining of overly Oriental-appearing eyelids and severe facial asymmetry. She had been visiting the Department of Pulmonary Medicine for bronchial asthma, with which she had been previously diagnosed. Her concern was mainly focused on the asymmetry of her eyes and the small vertical dimensions of her left palpebral fissure. The asymmetry involved her upper, middle, and lower face. Her left forehead and temporal region was retruded, while the right side was protruded. A definite disparity was observed in her eyebrow levels; the right side was elevated, while the left was descended, with lateral hooding. Radial expansion of the palpebral fissure was noted on her right side, while there was deflation and severe retrusion on her left side. The horizontal length was more deficient in her left eye, and the vertical dimension was far more deficient on her left side than on her right side. Midface hypoplasia and severe retrusion was noted along her zygomatic and malar area. Upslanting of her left oral commissure was noted, and it became more evident upon mouth-opening or smiling. Although she seemed to have normo-occlusion, she displayed mild occlusal canting upon biting a wooden tongue depressor (Fig. 1).

The initial encounter suggested the following possible differential diagnoses: hemifacial microsomia, progressive hemifacial atro- 


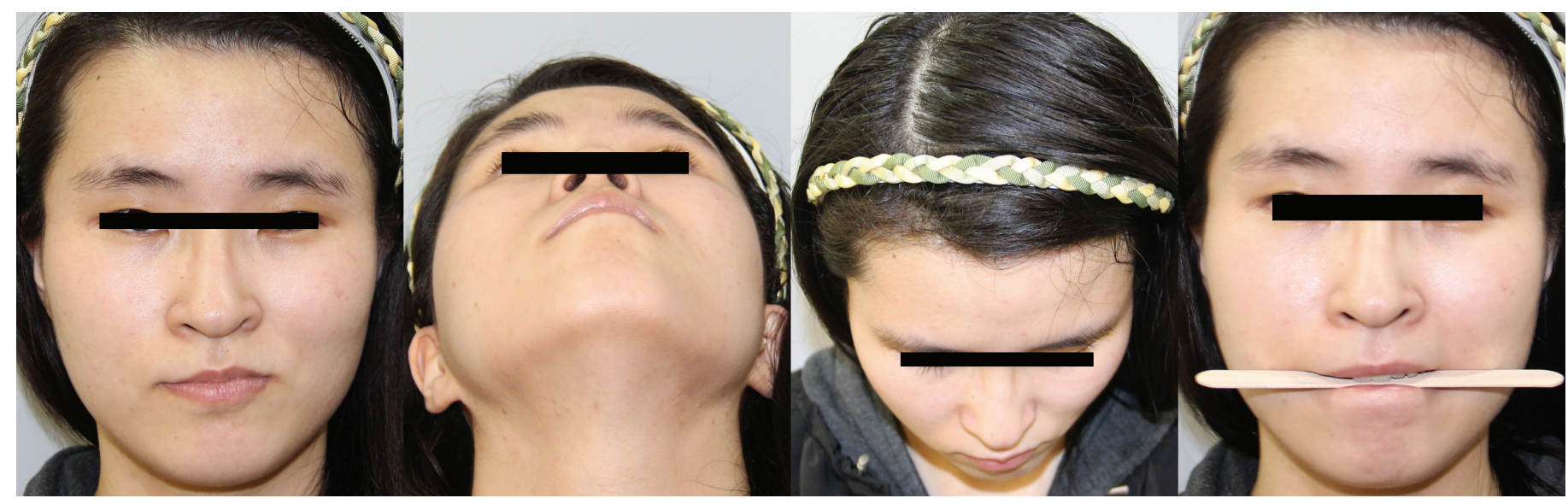

Fig. 1. Facial asymmetry involving the upper, middle, and lower third of the face, showing radial expansion of the right side and deflation of the left hemiface: left forehead retrusion, eyebrow slanting and lateral hooding, retrusion and deflation of the vertical dimension of the left palpebral fissure, a ptotic left upper eyelid, a hypoplastic midface with severe retrusion, tilting of the nasal axis toward the right side (nasal tip), upward mouth corner lifting and retrusion, and compensatory occlusal plane tilting.

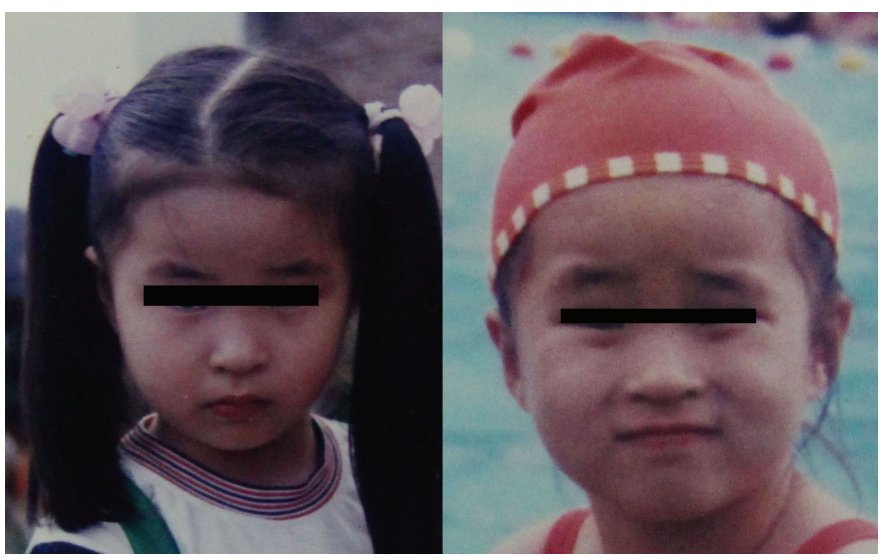

Fig. 2. Childhood photographs. Facial asymmetry was present even in childhood. The disease did not seem to have been progressive, as is characteristic of Parry-Romberg disease.

phy (namely, Parry-Romberg syndrome), unilateral coronal craniosynostosis, and lambdoidal craniosynostosis. Later, when the patient's mother brought an old photograph of the patient (Fig. 2), progression of the disease seemed far less likely, which led us to rule out Parry-Romberg disease. To rule out craniosynostosis, we had to determine whether her cranial suture lines were obliterated or remained intact.

Imaging studies were performed using facial 3-dimensional (3D) computed tomography (CT), and brain 3D CT, with the images reconstructed using algorithms. The reconstructed images revealed a clear and well-demarcated suture line in her skull. The intact sutures, without any obliteration, led us to make the definitive diagnosis of DP, instead of craniosynostosis. According to the DP severity classification proposed by Argenta, the subject fell into type
$4[1,2]$. The skull had a parallelogram shape and exhibited severe asymmetry. However, the asymmetry of the facial bone was much smaller than the facial asymmetry (Fig. 3 and 4).

In a comparison of the images shown in Fig. 1 and 3, the facial asymmetry was judged to be a problem of the facial soft tissue, and the authors proposed solving the problem by only soft tissue surgery, such as fat graft and eyelid surgery.

In order to restore static symmetry, the patient underwent bilateral double eyelidplasty using the non-incisional method (buried suture technique using 7-0 nylon), left levator palpebrae superioris and Müller's muscle aponeurosis plication using 7-0 nylon sutures (the levator palpebrae superioris muscle and Muller muscle aponeurosis were plicated $6 \mathrm{~mm}$ in length). In order to increase the horizontal dimensions of her palpebral fissures, bilateral epicanthoplasty using the skin redraping method was employed. To obtain volumetric expansion of her left hemiface, autologous microfat grafting was done from her bilateral thighs $(55 \mathrm{cc}$ of fat was harvested from both thighs and transplanted into the left cheek [20 cc] and the left forehead $[10 \mathrm{cc}])$. The remaining fat was cryopreserved for later secondary fat grafting at 1 month postoperatively. One month later, secondary fat grafting was performed, with $15 \mathrm{cc}$ transplanted into the left cheek and $10 \mathrm{cc}$ into the left forehead, resulting in a total implantation of $35 \mathrm{cc}$ of fat in the left cheek and $20 \mathrm{cc}$ in the left forehead.

The patient returned for a follow-up examination 4 months after surgery. The facial asymmetry was resolved and the patient was very satisfied with the results (Fig. 5).

\section{DISCUSSION}

Only in the last few decades have doctors been finally able to rec- 


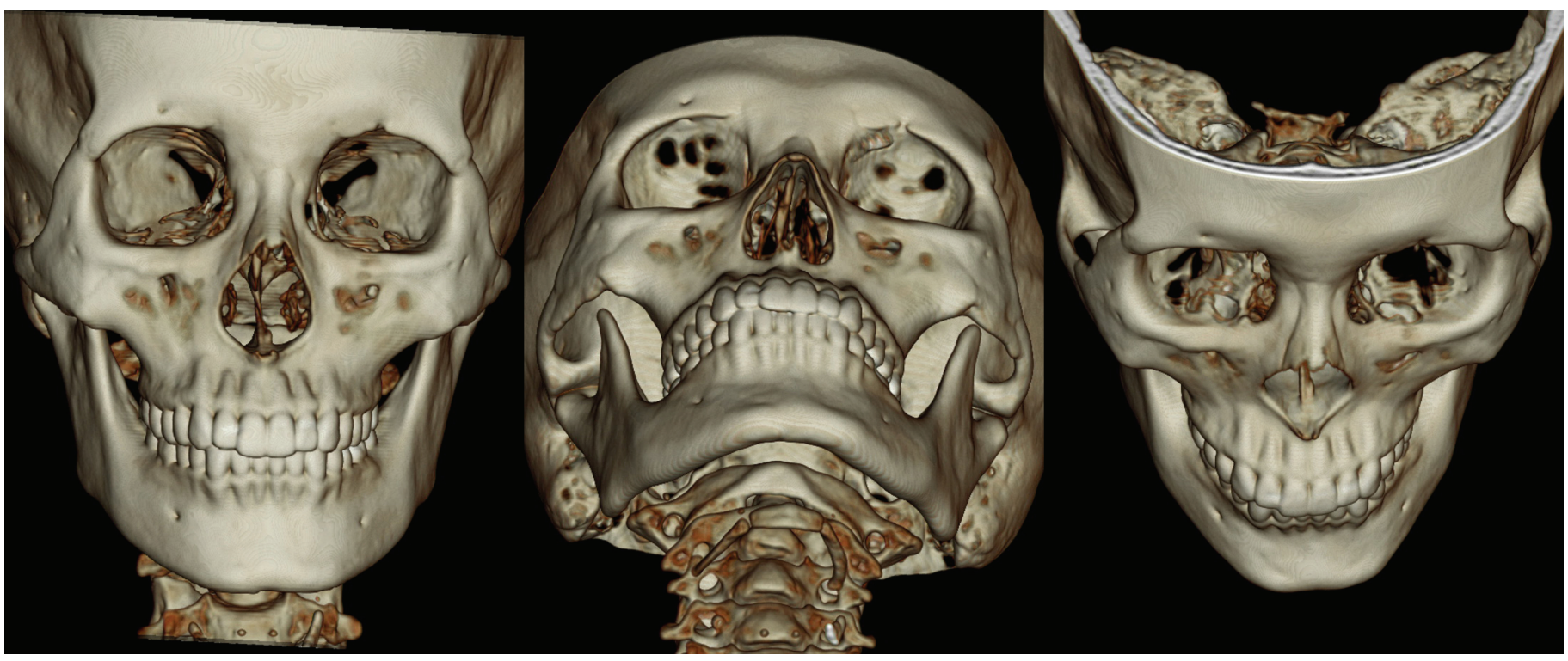

Fig. 3. Mild asymmetry of the facial skeleton was present. The vertical axis of the nasal bone was slightly tilted toward the right.

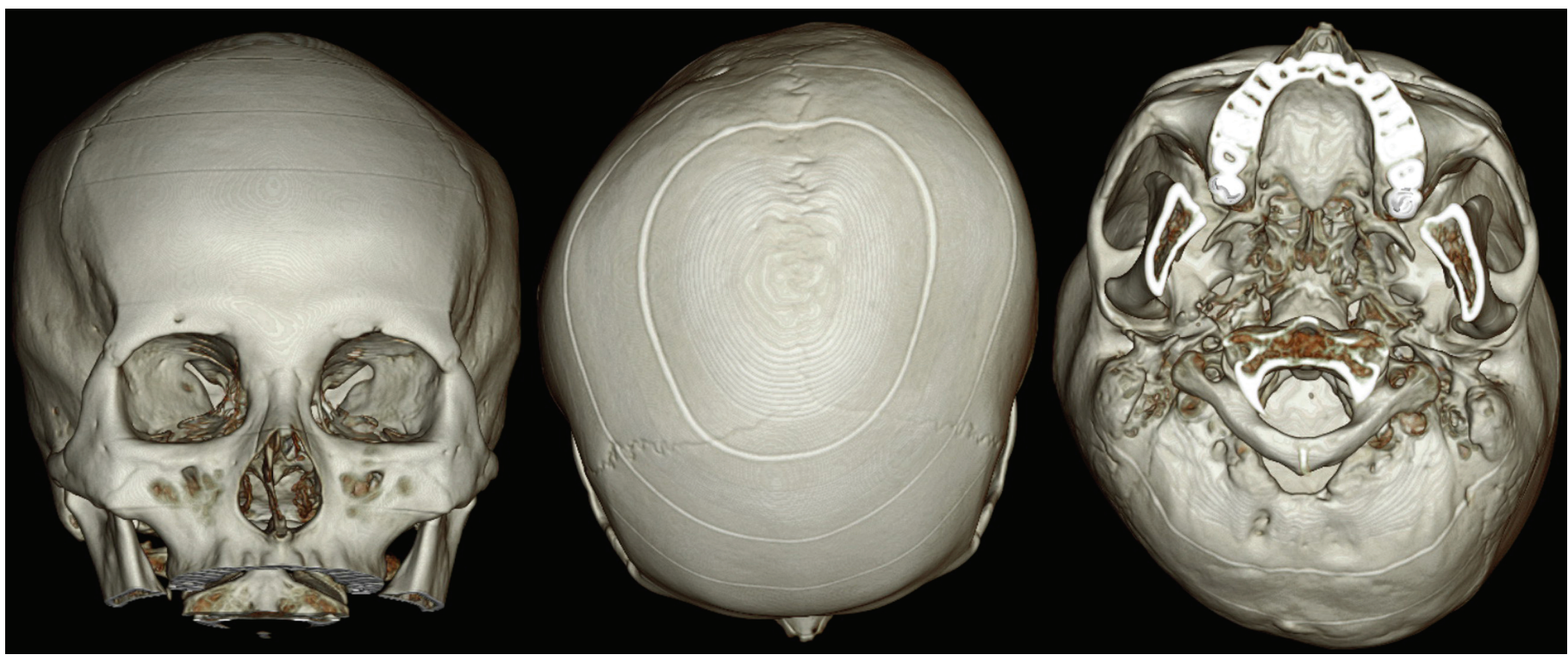

Fig. 4. All cranial suture lines were clear and intact without any obliteration. Severe indentation was noted in the right occipital area and depression was evident in the left forehead area, demonstrating the features of a parallelogram when the skull was seen from above.

ognize DP—also known as positional plagiocephaly—with reliability and accuracy. Its exact incidence and prevalence has not been established, with reported rates ranging from $5 \%$ to $45 \%$. In a study in 2012, the prevalence was estimated to be between $12 \%$ and $22 \%$ [3]. Because of similar facial features (a parallelogram-shaped skull, flattening of the affected occiput, ipsilateral frontal bossing, contralateral occiput bulging, and anterior shifting of the ipsilateral ear $[4,5]$ ), it was once often misdiagnosed as other congenital malformations, such as hemifacial microsomia, unilateral coronal craniosynostosis, or unilateral lambdoidal synostosis (ULS). The inci- dence skyrocketed in the United States after 1992 [6]. This sudden influx can be ascribed to the Back to Sleep campaign of the American Academy of Pediatrics that was conducted to reduce the rate of sudden infant death syndrome (SIDS) [7]. After this campaign was conducted, the SIDS rate plummeted by more than $40 \%$ [8].

Several methods for diagnosing DP and classifying its severity have been proposed, including the Hutchinson severity scale using the diagonal difference (DD) (mild, $3 \mathrm{~mm}<\mathrm{DD}<10 \mathrm{~mm}$; moderate, $10 \mathrm{~mm}<\mathrm{DD}<12 \mathrm{~mm}$; and severe, $\mathrm{DD}>12 \mathrm{~mm}$ ) [5], anthropometric caliper measurements (by Willbrand et al. 2012), the cra- 


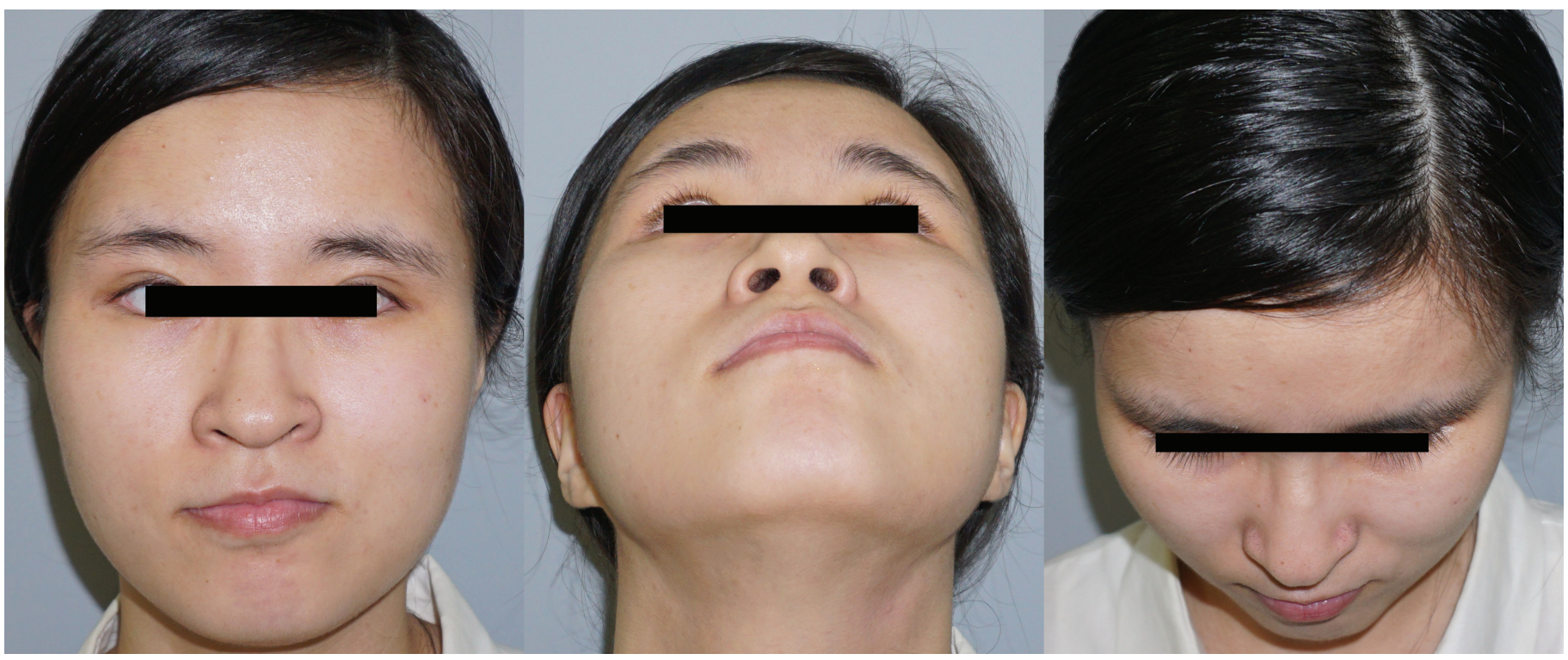

Fig. 5. Three-month postoperative photographs show an improved facial contour and improved facial asymmetry. The enlargement of the eyes through eyelidplasty and the plication of the levator palpebrae superioris muscle aponeurosis and Müller's muscle aponeurosis compensated for the different palpebral size of the left and the right eyes. Volumetric inflation of the left hemiface through a fat graft enhanced her overall appearance.

nial vault asymmetry index, plagiocephalometry using a flexicurve ruler, and radiographic imaging such as CT scans [9]. Although there is currently no established way to quantify the severity of DP accurately, some attempts have been made to categorize its severity [7]. In 2004, Argenta et al. [1] proposed a severity classification system that classifies DP into 5 different types [2]. The subject in this report matched the criteria for type 4 (posterior asymmetry + ear malposition + forehead asymmetry + facial asymmetry) [7]. The risk factors for DP can be divided into perinatal risk factors and maternal risk factors, including a low education level, young maternal age, and bottle feeding. Perinatal/birth risk factors include first-born infants [10], supine positioning, premature birth, prolonged intubation, male gender, intrauterine restriction in multiple gestation pregnancies (fraternal twins $>$ maternal twins) [8], breech position in uterus, primiparous mothers, assisted delivery, maternal age $>35$ years, birth trauma, congenital anomalies, a low activity level, delayed milestones, bottle feeding, and prolonged labor $[7,11]$. The patient in our study was an identical twin, corresponding to a known risk factor for DP. In utero, the lower fetus is engaged in the maternal pelvis and bears the weight of the second fetus [8]. The reason why preterm $(<32$ gestational weeks) infants have a higher risk is explained by the fact that they are subjected to extrauterine gravity at an early stage [3]. DP typically is more common on the right side than the left.

DP is commonly associated with diseases that may be the result of the disease or an aggravating factor, such as congenital muscular torticollis (CMT), strabismus, or astigmatism $[7,12]$. CMT is a risk factor for DP that has been well documented in the literature [8].
The importance of carefully looking for other associated diseases when DP is diagnosed should be underscored. Fortunately, in our case, the patient did not have any signs of CMT or ophthalmologic abnormalities. CMT may be present in as many as $90 \%$ of affected persons [12], but is often overlooked, and the diagnosis of CMT prior to the diagnosis of DP is made in as few as $24 \%$ of affected individuals $[7,12]$. The stiffness of the contralateral sternocleidomastoid muscle seen in most DP patients is generally regarded as mere stiffness, rather than true atrophy or fibrosis of the muscle [12].

Other abnormalities associated with DP include auditory processing delays and delays in cognitive and psychomotor development $[4,12]$. The differential diagnosis of DP includes central nervous system lesions, vertebral deformities, craniosynostosis, and brachycephaly.

Unlike other congenital disorders that may be candidates in the differential diagnosis, the treatment for DP (or positional plagiocephaly) is helmet therapy and position-changing postural therapy. A considerable amount of the literature presents helmet therapy as the most effective treatment modality when early intervention is implemented [5]. Helmet therapy corrects cranial asymmetry and ear position, while counterpositioning therapy corrects only ear shifting [5]. The rationale behind early therapy is that it harnesses the plasticity of the skull and it can be initiated as early as 4 to 6 months postnatally [13].

Craniosynostosis, which has an overall incidence of 1 out of every 2000 births [7], is a congenital abnormality characterized by the premature closure of one or more of the cranial suture lines, which may bring about life-threatening complications if left un- 
treated, such as increased intracranial pressure, which may in turn result in blindness; mental retardation; visual, hearing, or speech deficits; airway obstruction; and death [7]. This possibility warrants quick surgical intervention, and surgery remains the mainstay therapy for most cases of craniosynostosis [9]. The features of craniosynostosis are strikingly similar to those of DP. For unilateral coronal craniosysnostosis (UCS), the following features are observed: ipsilateral frontal bone flattening with elevation and retrusion of the superior orbital rim, forehead bossing, anterior and inferior displacement of the contralateral superior orbital rim, deviation of the nasal root toward the fusion, and rotation of the middle and lower face to the contralateral side [14]. For ULS, the characteristic features are skull tilting, mastoid bossing, and a parallelogram shape with or without asymmetric cranial height. The only feature absent in DP is mastoid bossing (in a typical case of DP, the mastoid skull base is symmetrical [12]), and this could be an important clue for differentiating among these conditions [7]. Although ULS often presents with the ipsilateral ear displaced in the posterior and inferior directions, while DP presents with the ipsilateral ear displaced in the anterior direction, differentiating these 2 diseases based on ear position seems unreliable [10]. The treatments for both UCS and ULS are surgical: fronto-orbital advancement or strip craniectomy [14] and barrel-stave craniectomy, respectively.

Hemifacial microsomia is also known as lateral facial dysplasia, first and second branchial arch syndrome, oral-mandibular-auricular syndrome, otomandibular dysostosis, and craniofacial microsomia (CFM). Its clinical features involve hypoplasia of the maxillary, mandibular, and auricular areas. Pruzansky classified the disease according to the extent of mandibular deformity, stating that condylar involvement is a pathognomonic sign of hemifacial microsomia. The severe form of DP can mimic hemifacial microsomia, and a diagnostic workup is necessary to differentiate between these conditions. However, in terms of the urgency of the intervention, hemifacial microsomia is less of an emergency than craniosynostosis.

\section{CONCLUSION}

Despite the high prevalence of DP patients, many do not receive appropriate treatment (helmet therapy). The case above shows that facial asymmetry without bone asymmetry can occur in a DP patient who missed the treatment window. In such cases, the facial bone asymmetry is not severe, and dramatic aesthetic improvements are possible with soft tissue handling and without massive bone surgery. This also provided a high level of satisfaction to the patient. The authors consider DP patients similar to the patient described in this case to be good candidates for aesthetic surgery, which can provide great benefits with minor invasiveness. For this reason, we wanted to share our experiences with the readers of this journal.

\section{PATIENT CONSENT}

Patients provided written consent for the use of their images.

\section{REFERENCES}

1. Argenta L, David L, Thompson J. Clinical classification of positional plagiocephaly. J Craniofac Surg 2004;15:368-72.

2. Spermon J, Spermon-Marijnen R, Scholten-Peeters W. Clinical classification of deformational plagiocephaly according to Argenta: a reliability study. J Craniofac Surg 2008;19:664-8.

3. Nuysink J, van Haastert IC, Eijsermans MJ, et al. Prevalence and predictors of idiopathic asymmetry in infants born preterm. Early Hum Dev 2012;88:387-92.

4. Collett BR, Aylward EH, Berg J, et al. Brain volume and shape in infants with deformational plagiocephaly. Childs Nerv Syst 2012;28:108390.

5. Kim SY, Park MS, Yang JI, et al. Comparison of helmet therapy and counter positioning for deformational plagiocephaly. Ann Rehabil Med 2013;37:785-95.

6. Steinbok P, Lam D, Singh S, et al. Long-term outcome of infants with positional occipital plagiocephaly. Childs Nerv Syst 2007;23:1275-83.

7. Unwin S, Dika C. Deformational plagiocephaly: a focus on prevention. J Nurse Pract 2017;13:162-9.

8. Littlefield TR, Kelly KM, Pomatto JK, et al. Multiple-birth infants at higher risk for development of deformational plagiocephaly: II. is one twin at greater risk? Pediatrics 2002;109:19-25.

9. Siegenthaler MH. Methods to diagnose, classify, and monitor infantile deformational plagiocephaly and brachycephaly: a narrative review. J Chiropr Med 2015;14:191-204.

10. Pogliani L, Mameli C, Fabiano V, et al. Positional plagiocephaly: what the pediatrician needs to know. A review. Childs Nerv Syst 2011;27: 1867-76.

11. Cabrera-Martos I, Valenza MC, Benitez-Feliponi A, et al. Clinical profile and evolution of infants with deformational plagiocephaly included in a conservative treatment program. Childs Nerv Syst 2013;29:1893-8.

12. Losee JE, Mason AC. Deformational plagiocephaly: diagnosis, prevention, and treatment. Clin Plast Surg 2005;32:53-64.

13. Mortenson P, Steinbok P, Smith D. Deformational plagiocephaly and orthotic treatment: indications and limitations. Childs Nerv Syst 2012; 28:1407-12.

14. Levy RL, Rogers GF, Mulliken JB, et al. Astigmatism in unilateral coronal synostosis: incidence and laterality. J AAPOS 2007;11:367-72. 